
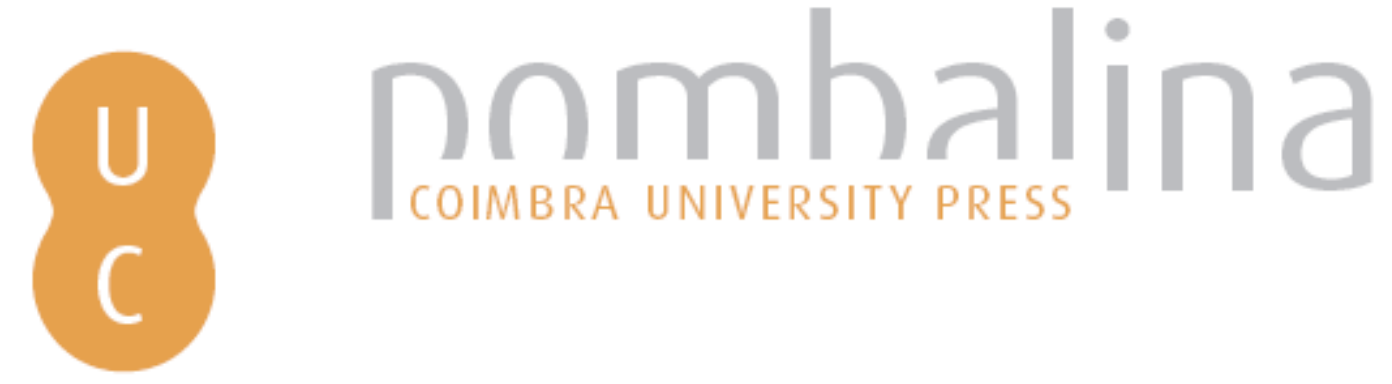

\title{
Contributos sedimentológicos e geoquímicos para a modelação dos sistemas petrolíferos portugueses: as séries jurássicas ricas em matéria orgânica da Bacia Lusitânica (Portugal)
}
Autor(es): $\quad$ Silva, Ricardo; Duarte, Luís; Azerêdo, Ana; Mendonça Filho, João
Publicado por: Imprensa da Universidade de Coimbra; Laboratório de Radioactividade Natural da Universidade de Coimbra

URL persistente:

URI:http://hdl.handle.net/10316.2/36318

DOI:

DOI:http://dx.doi.org/10.14195/978-989-26-1009-2_29

Accessed : $\quad$ 26-Apr-2023 14:48:29

A navegação consulta e descarregamento dos títulos inseridos nas Bibliotecas Digitais UC Digitalis, UC Pombalina e UC Impactum, pressupõem a aceitação plena e sem reservas dos Termos e Condições de Uso destas Bibliotecas Digitais, disponíveis em https://digitalis.uc.pt/pt-pt/termos.

Conforme exposto nos referidos Termos e Condições de Uso, o descarregamento de títulos de acesso restrito requer uma licença válida de autorização devendo o utilizador aceder ao(s) documento(s) a partir de um endereço de IP da instituição detentora da supramencionada licença.

Ao utilizador é apenas permitido o descarregamento para uso pessoal, pelo que o emprego do(s) título(s) descarregado(s) para outro fim, designadamente comercial, carece de autorização do respetivo autor ou editor da obra.

Na medida em que todas as obras da UC Digitalis se encontram protegidas pelo Código do Direito de Autor e Direitos Conexos e demais legislação aplicável, toda a cópia, parcial ou total, deste documento, nos casos em que é legalmente admitida, deverá conter ou fazer-se acompanhar por este aviso.

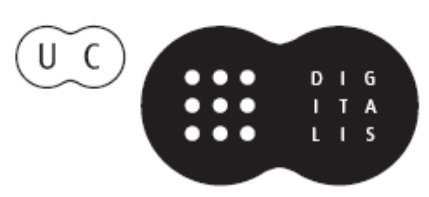




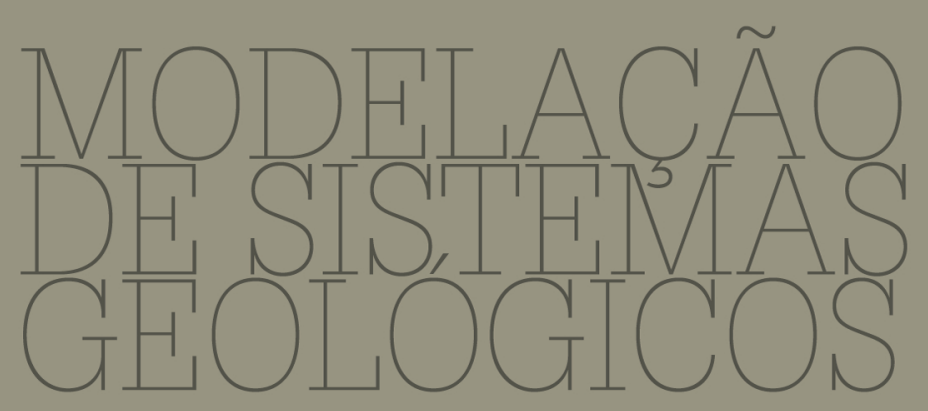

Homenagem ao Professor Doutor Manuel Maria Godinho

L.J.P.F. Neves, A.J.S.C. Pereira,

C.S.R. Gomes, L.C.G. Pereira,

A.O. TAVARES

IMPRENSA DA UNIVERSIDADE DE COIMBRA

CoImBra University Press 


\title{
Contributos sedimentológicos e geoquímicos para a modelação dos sistemas petrolíferos portugueses: as séries jurássicas ricas em matéria orgânica da Bacia Lusitânica (Portugal)
}

\author{
Ricardo Silva ${ }^{1}$; Luís Duarte ${ }^{1}$; Ana Azerêdo ${ }^{2}$; João Mendonça Filho ${ }^{3}$ \\ ${ }^{1}$ Departamento de Ciências da Terra e IMAR-CMA, Faculdade de Ciências e Tecnologia. Universidade \\ de Coimbra, Largo Marquês de Pombal, 3000-272 Coimbra, PORTUGAL. \\ ricardo.silva@student.dct.uc.pt, Iduarte@dct.uc.pt \\ ${ }^{2}$ Universidade de Lisboa, Faculdade de Ciências, Departamento de Geologia e Centro de Geologia, \\ Campo Grande, 1749-016, Lisboa, PORTUGAL. acazeredo@fc.ul.pt \\ ${ }^{3}$ Departamento de Geologia, Instituto de Geociências, Universidade Federal do Rio de Janeiro. Cidade \\ Universitária, CEP: 21949-900, Ilha do Fundão, Rio de Janeiro, BRASIL. graciano@geologia.ufrj.br
}

Palavras-chave: Rocha geradora, Formação de Vale das Fontes, Formação de Cabaços, Jurássico, Bacia Lusitânica

\section{Resumo}

A elaboração de modelos sedimentológicos preditivos é uma das principais ferramentas na prospecção de petróleo. No entanto, no que respeita aos sistemas carbonatados, a concepção de modelos geológicos coerentes é complexa, devido à intrincada relação entre os diversos parâmetros físicos, químicos, geológicos e biológicos que controlam a deposição nesses ambientes. O objectivo deste trabalho é o de apresentar uma caracterização sumária de dois dos mais importantes intervalos estratigráficos potencialmente geradores de petróleo da Bacia Lusitânica: o membro Margo-calcários com níveis betuminosos da Formação de Vale das Fontes (Pliensbaquiano) e a Formação de Cabaços (Oxfordiano inferior?/médio). Os estudos em progresso, em especial sobre a matéria orgânica, deverão conduzir a trabalhos de modelação visando aperfeiçoar o conhecimento da evolução lateral e temporal dos ambientes deposicionais e da geração, maturação e migração de hidrocarbonetos relacionados com os referidos intervalos estratigráficos.

Key-words: Source rock, Vale das Fontes Formation, Cabaços Formation, Jurassic, Lusitanian Basin

\section{Abstract}

The elaboration of predictive sedimentological models is one of the main tools in petroleum prospection. However, regarding carbonate systems, building-up coherent geological models is complex due to the intricate relationship between the several physical, chemical, 
geological and biological factors that control the deposition in those environments. The objective of this work is to present a brief characterization of two of the most important Jurassic potential source-rocks in the Lusitanian Basin: the Marly limestones with organicrich facies member of the Vale das Fontes Formation (Pliensbachian) and the Cabaços Formation (Early?/Middle Oxfordian). Ongoing studies, particularly on organic matter, will likely lead to modelling works aiming at improving current knowledge on the lateral and temporal evolution of the depositional environments and hydrocarbon generation, maturation and migration related to those stratigraphical intervals.

\section{Introdução}

A prospecção de hidrocarbonetos assenta num vasto conjunto de ferramentas, sendo uma delas a elaboração de um modelo evolutivo da bacia que permita a dedução das mais diversas propriedades dos corpos rochosos presentes numa determinada área em prospecção. No entanto, a concepção de modelos com significado geológico relativos aos sistemas sedimentares carbonatados é difícil, uma vez que estes implicam um conhecimento alargado sobre os vários factores físicos, químicos, geológicos e biológicos que governam a sedimentação nestes ambientes de deposição.

No registo jurássico da Bacia Lusitânica (BL) são reconhecidos vários intervalos ricos em matéria orgânica. Destes, apenas dois têm distribuição bacinal e são reconhecidos como rochas potencialmente geradoras de petróleo. $\mathrm{O}$ primeiro corresponde ao membro Margo-calcários com níveis betuminosos (mb MCNB) da Formação de Vale das Fontes (Pliensbaquiano) e o segundo à Formação de Cabaços (Oxfordiano inferior?/médio) (ver por exemplo, Azerêdo et al., 2002; Oliveira et al., 2006; Duarte et al., 2010a,b; Silva et al., 2010a). Este trabalho tem como objectivo apresentar uma caracterização sumária das duas unidades litológicas referidas, desde o seu contexto e dinâmica sedimentar ao conteúdo em matéria orgânica e parâmetros associados, tendo como base os estudos de detalhe actualmente em curso.

\section{Enquadramento geológico genérico dos dois intervalos em estudo}

A BL é uma pequena bacia de direcção aproximadamente Norte-Sul, situada no lado ocidental do Maciço Ibérico (Figura 1). A sua origem está relacionada com a abertura do Oceano Atlântico e apresenta várias semelhanças com outras bacias vizinhas (por exemplo, Wilson et al., 1989; 
Azerêdo et al., 2002; Duarte et al., 2010b e referências contidas nestes trabalhos).
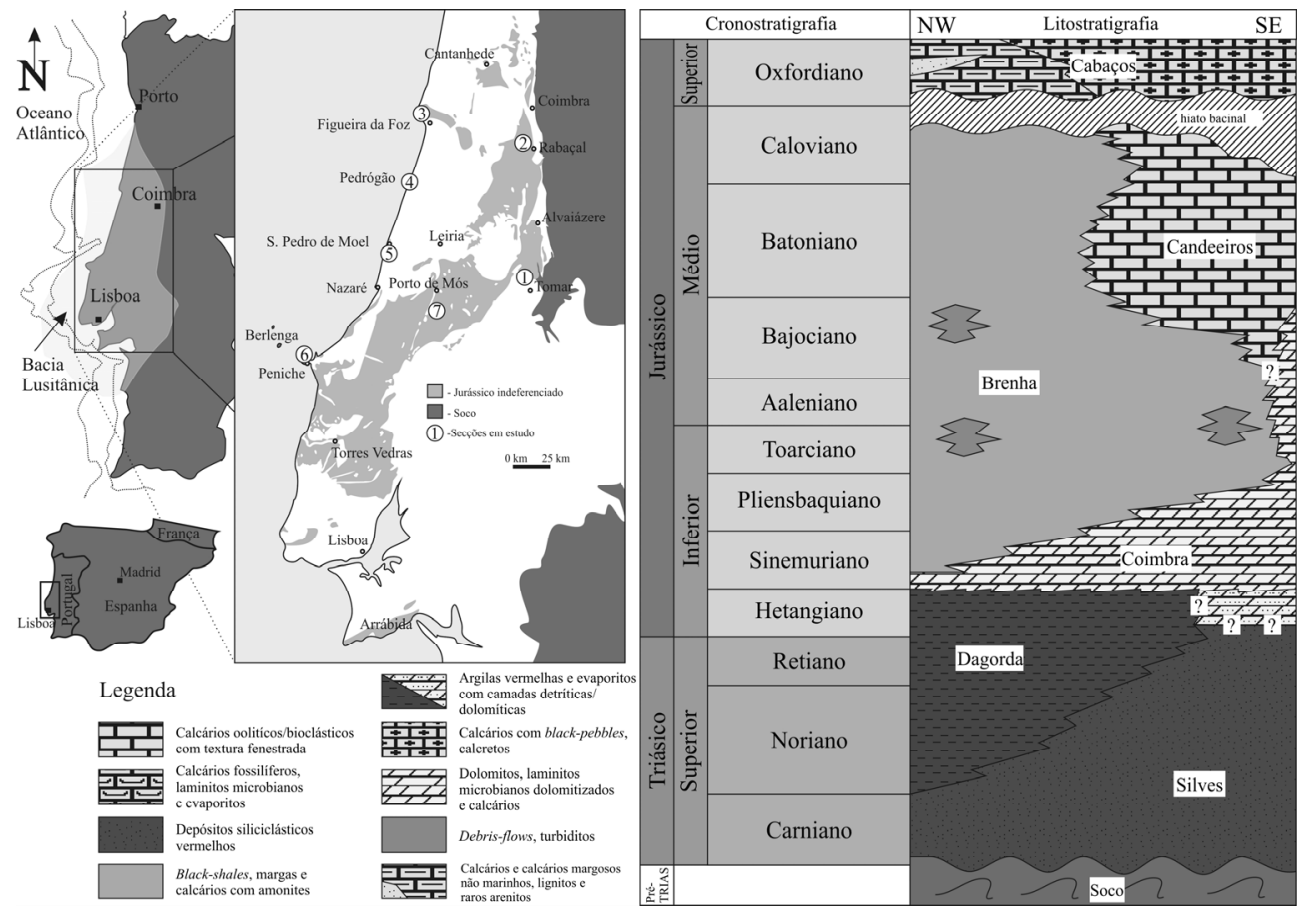

Figura 1. Mapa geológico simplificado, referente aos afloramentos do Jurássico na Bacia Lusitânica e esquema litostratigráfico genérico para o intervalo Triásico Superior-Jurássico Superior; modificado de Azerêdo et al. (2002).

A evolução triásico-jurássica da BL consiste em duas grandes etapas de sedimentação limitadas por descontinuidades, correspondendo aos períodos de tempo Triásico Superior-Jurássico Médio e Jurássico SuperiorCretácico Inferior (Wilson et al., 1989). As unidades de Silves e de Dagorda marcam o início da sedimentação na bacia e são sobrepostas pela Formação de Coimbra e os grupos informais Brenha e Candeeiros, representando o início e desenvolvimento de um sistema deposicional do tipo rampa carbonatada, aberta para noroeste (Figura 1). O primeiro intervalo em estudo, o mb MCNB da Formação de Vale das Fontes, é incluído no grupo Brenha e é de idade topo de Ibex-Margaritatus (Pliensbaquiano). Corresponde a um conjunto de alternâncias margo-calcárias de natureza hemipelágica, com um abundante registo de macrofósseis bentónicos e nectónicos (Duarte e Soares, 2002; Duarte, 2007; Duarte et al., 2010b). 
A passagem Jurássico Médio-Superior corresponde a um hiato bacinal, abrangendo o Caloviano Superior-Oxfordiano Inferior, embora, pontualmente e em regiões mais a leste, possa abranger até o Batoniano superior. Esta desconformidade é precedida por uma regressão forçada complexa, resultando em variações litológicas bruscas (Azerêdo et al., 2002). Os primeiros sedimentos do Jurássico Superior pertencem à Formação de Cabaços (Figura 1) e correspondem ao desenvolvimento de sistemas deposicionais do tipo lacustre, lagunar e marinho raso (Azerêdo et al., 2002; Azerêdo e Cabral, 2004; Azerêdo et al., 2010a,b).

\section{Jurássico Inferior: Mb Margo-calcários com níveis betuminosos}

$\mathrm{O} \mathrm{mb} \mathrm{MCNB}$, quando comparado com as unidades enquadrantes, é caracterizado por um aumento dos litótipos margosos e pela ocorrência das fácies ricas em matéria orgânica, particularmente bem desenvolvidas no sector oeste (Duarte e Soares, 2002; Duarte et al., 2010b) (Figura 2).

Com base em diversos critérios, e embora a variação litológica seja pequena, é possível distinguir três domínios no sector norte da BL (ver por exemplo, Duarte e Soares, 2002; Duarte et al., 2004, Duarte, 2007; Silva et al., 2010b). A oeste (Peniche, S. Pedro de Moel e Brenha) e correspondendo ao sector mais distal, a principal característica do mb MCNB é a abundância das fácies ricas em matéria orgânica e os seus elevados valores de COT (cerca de 15-20\%), incluindo até mesmo vários níveis de verdadeiros blackshales (Duarte et al., 2010a,b; Silva et al., 2010c).

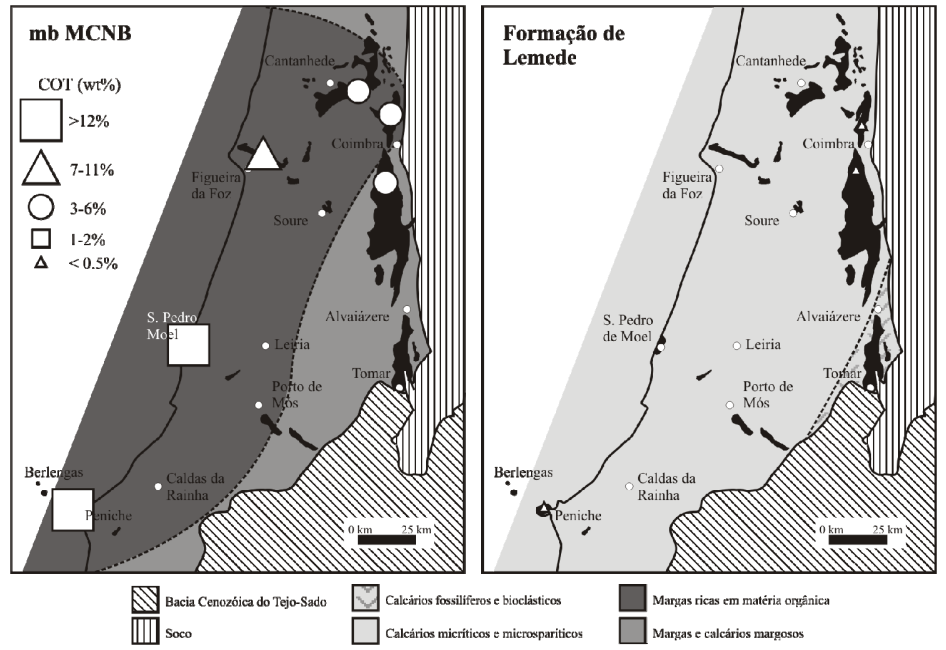

Figura 2. Mapas de distribuição de fácies e variação lateral de COT para o mb MCNB e unidade subjacente, a Formação de Lemede (modificado de Duarte et al., 2010b). 
São também abundantes os macrofósseis nectónicos (amonites e belemnites) e bentónicos (braquiópodes, bivalves, equinodermes e gastrópodes). No domínio centro-leste (Rabaçal), a sedimentação tem um carácter intermédio e distingue-se pela ocorrência significativa de nautilóides, pela presença de pequenos montículos mud-wacke-packstone (associados com nautilóides na base ou com grandes Litoceratídeos no topo da secção) e restos de animais de maior porte (ictiossáurios?). Localmente são observadas fácies ricas em matéria orgânica. $\mathrm{O}$ sector sudeste (Tomar) representa o ambiente de menor profundidade estudado. As suas características são a ausência das fácies ricas em matéria orgânica e o aumento da expressão dos macrofósseis bentónicos (nomeadamente dos braquiópodes e bivalves) e de fácies bioclásticas. As observações palinofaciológicas e o estudo dos biomarcadores relacionados com proveniência (Figura 3) mostram que a matéria orgânica particulada consiste numa mistura variável de componentes marinhos e continentais preservados num ambiente deposicional marinho com condições redox variáveis (Silva et al., 2010c).

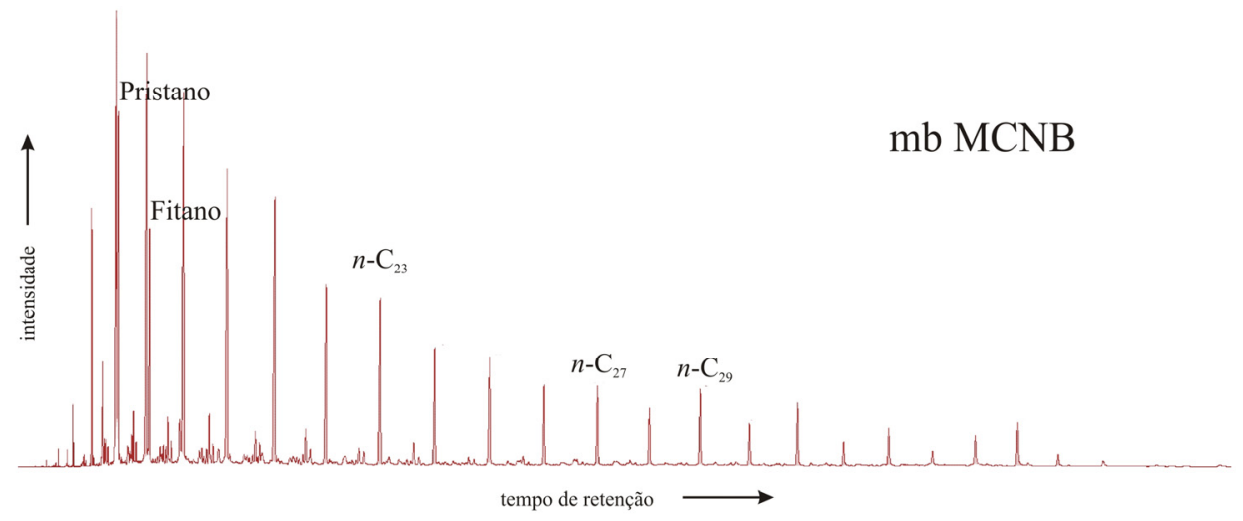

Figura 3. Cromatograma $(\mathrm{m} / \mathrm{z} 85)$ de uma amostra do mb MCNB.

\section{Jurássico Superior: Formação de Cabaços}

A Formação de Cabaços (Oxfordiano inferior?/médio) representa os primeiros sedimentos de idade Jurássico Superior na BL. O seu registo em afloramento é relativamente reduzido e o seu reconhecimento de campo implica bom conhecimento da unidade, pois é condicionado pela ausência de qualquer (macro)fóssil-índice (ver em Azerêdo et al., 2010a,b, a mais recente observação, em afloramento, desta unidade).

Esta unidade apresenta uma grande dinâmica sedimentar. A norte, na base da secção do Cabo Mondego, observam-se fácies de carácter misto 
entre o siliciclástico e o carbonatado, possivelmente correspondendo a uma primeira incursão deltaica e ao desenvolvimento de pequenas baías de água doce a salobra (Wright, 1985). Um pouco mais a sul e ao longo da costa, em Pedrógão, a parte da série lateralmente equivalente ao referido acima para o Cabo Mondego, tem um carácter parálico, com o desenvolvimento de sistemas lacustres semi-permanentes que se tornam persistentes para sudeste e com claras evidências de exposição (Azerêdo et al., 2002; Azerêdo e Cabral, 2004) (Figura 4). Para o topo, a sucessão tende a apresentar um carácter mais marinho (ex. Azerêdo et al., 2002), tornando-se marinho franco na unidade sobrejacente, a Formação de Montejunto, embora pontualmente se observem fácies proximais do tipo peritidal (ex. Azerêdo e Wright, 2004; Wright e Azerêdo, 2006).

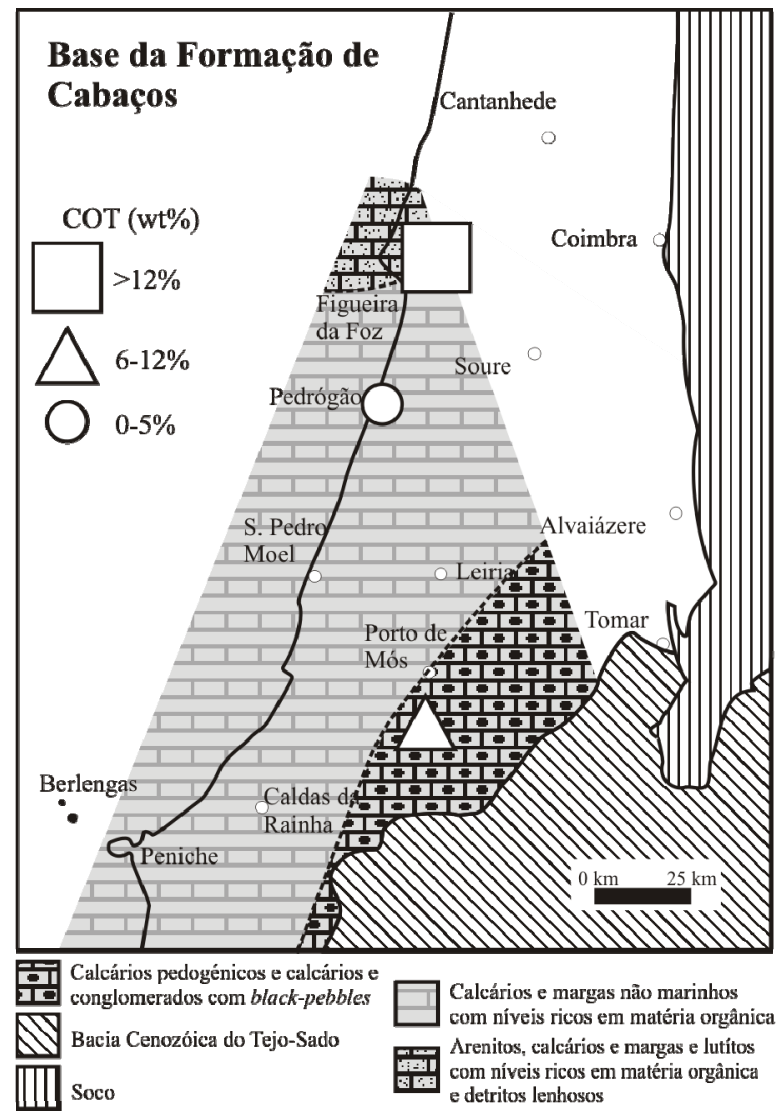

Figura 4. Mapa genérico de distribuição de fácies (de acordo com Azerêdo et al., 2002) e COT máximo para a base da Formação de Cabaços. 
O valor máximo de $30,7 \%$ de COT na Formação de Cabaços foi determinado no Cabo Mondego, atingindo 11,6\% no Maciço Calcário Estremenho (Vale de Ventos) e 4,7\% em Pedrógão. Os estudos palinofaciológicos em curso nestes locais mostram que a matéria orgânica particulada é predominantemente continental (fitoclastos, pólen e esporos), pontuada por eventos menores de influência marinha (por exemplo, ocorrência de raras algas Prasinophyta e quistos de dinoflagelados). Alguns dos níveis analisados possuem uma importante contribuição de outros tipos de componentes, como matéria orgânica amorfa ou algal de água doce (Silva et al., 2010a e ver também Barron e Azeredo, 2003 para um maior detalhe sobre os estudos palinológicos). Os biomarcadores relacionados com proveniência são consistentes com as palinofácies (Figura 5).

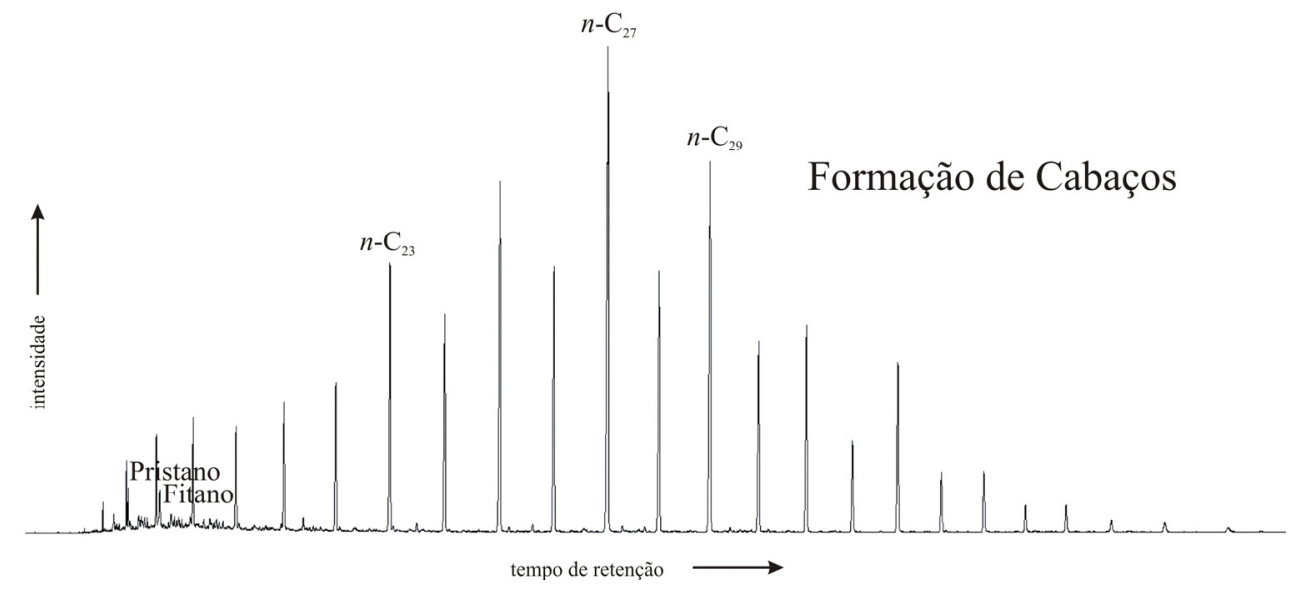

Figura 5. Cromatograma $(\mathrm{m} / \mathrm{z} 85)$ de uma amostra da Formação de Cabaços.

\section{Considerações Finais}

$\mathrm{Na} \mathrm{BL}$, o mb MCNB e a Formação de Cabaços correspondem aos dois intervalos estratigráficos com extensão bacinal potencialmente geradores de petróleo. A intersecção dos diversos parâmetros sedimentológicos e geoquímicos em estudo, com recurso paralelo a quadros biostratigráficos detalhados e/ou a observações de campo de alta resolução, permitirá uma reconstituição bastante fina das condições paleoceanográficas vigentes durante a sedimentação daquelas unidades e o aperfeiçoamento dos respectivos modelos sedimentares. 


\title{
Agradecimentos
}

\author{
Agradece-se à FCT-MCTES pelo suporte financeiro providenciado a \\ Ricardo Silva, através de uma Bolsa de Doutoramento. Este trabalho é uma \\ contribuição para o projecto PTDC/CTE-GIX/098968/2008, financiado pela \\ FCT-MCTES.
}

\section{Bibliografia}

Azerêdo A.C., Cabral, M-C. (2004) - Bio-sedimentary signatures of high-frequency salinity/subaerial exposure changes: Examples from the Oxfordian of Portugal (Cabaços Formation). Rivista Italiana di Paleontologia e Stratigrafia; 110 (1), 231-238.

Azerêdo A.C., Cabral, M.C., Martins, M.J.F., Loureiro, I.M., Inês, N. (2010a) - Nota preliminar sobre a ocorrência da Formação de Cabaços (Oxfordiano) na região da Serra do Bouro (Caldas da Rainha). Resumos estendidos do VIII Congresso Nacional de Geologia. e-Terra, 2010a; 21 (6), 4 p..

Azerêdo, A. C.; Cabral, M. C.; Martins, M.J.; Loureiro, I. M. \& Inês, N. (2010b) (in press) Estudo estratigráfico dum novo afloramento da Formação de Cabaços (Oxfordiano) na região da Serra do Bouro (Caldas da Rainha). Comunicações Geológicas, 97.

Azerêdo, A.C., Wright, V.P., Ramalho, M.M. (2002) - The Middle-Late Jurassic forced regression and disconformity in central Portugal: eustatic, tectonic and climatic effects on a carbonate ramp system. Sedimentology; 49, 1339-1370.

Azerêdo, A.C., Wright, V.P. (2004) - Multi-scale signatures and events in carbonate systems (Middle to early Upper Jurassic, Lusitanian Basin). In: Duarte, L.V., Henriques, M.H. (Eds.), Carboniferous and Jurassic Carbonate Platforms of Iberia. $23^{\text {rd }}$ IAS Meeting of Sedimentology, Field Trip Guide Book, Coimbra; 1, 75-91.

Barrón, E., Azerêdo, A.C. (2003) - Palynology of the Jurassic (Callovian-Oxfordian) succession from Pedrógão (Lusitanian Basin, Portugal). Palaeoecological and palaeobiogeographical aspects. Neues Jahrbuch für Geologie und Paläontologie, Abhandlungen; 227, 259-286.

Duarte, L.V. (2007) - Lithostratigraphy, sequence stratigraphy and depositional setting of the Pliensbachian and Toarcian series in the Lusitanian Basin (Portugal). In: R. B. Rocha (ed). The Peniche section (Portugal). Contributions to the definition of the Toarcian GSSP. International Subcommission on Jurassic Stratigraphy 2007, 17-23.

Duarte, L.V., Silva, R.L., Mendonça Filho, J.G., Oliveira, L.C. (2010a) - Evidências geoquímicas do Jurássico Inferior de Peniche (Bacia Lusitânica, Portugal): Estado de arte e perspectivas futuras. X Congresso de Geoquímica dos Países de Língua Portuguesa, XVI Semana de Geoquímica, Memórias; 15, 3-24.

Duarte, L.V., Silva, R.L. Oliveira, L.C.V., Comas-Rengifo, M.J., Silva, F. (2010b) - Organic-Rich facies in the Sinemurian and Pliensbachian of the Lusitanian Basin, Portugal: Total organic carbon distribution and relation to transgressive-regressive facies cycles. Geologica Acta; 8 (3), 325-340.

Duarte, L.V., Soares, A.F. (2002) - Litostratigrafia das series margo-calcárias do Jurássico Inferior da Bacia Lusitânica (Portugal). Comunicações do Instituto Geológico e Mineiro; 89: 135-154.

Duarte, L.V., Wright, V.P., López, S.F., Elmi, S., Krautter, M., Azerêdo, A.C., Henriques, M.H., Rodrigues, R., Perilli, N. (2004) - Early Jurassic carbonate evolution in the Lusitanian Basin (Portugal): facies, sequence stratigraphy and cyclicity. In: Duarte, L.V., Henriques, M.H. (Eds.), Carboniferous and Jurassic Carbonate Platforms of Iberia. $23^{\text {rd }}$ IAS Meeting of Sedimentology, Field Trip Guide Book, Coimbra; 1, 45-71.

Oliveira, L.C.V., Rodrigues, R., Duarte, L.V., Lemos, V. (2006) - Avaliação do potencial gerador de petróleo e interpretação paleoambiental com base em biomarcadores e isótopos estáveis do 
carbono da seção Pliensbaquiano-Toarciano inferior (Jurássico inferior) da região de Peniche (Bacia Lusitânica, Portugal). Boletim de Geociências da Petrobras; 14 (2), 207-234.

Silva, R.L., Azerêdo, A.C., Mendonça Filho, J.G., Duarte, L.V., Cabral, M.C. (2010a) - The MiddleUpper Jurassic transition in the Lusitanian Basin (Portugal): preliminary evaluation of TOC, Sulphur, $\delta^{13} \mathrm{C}$ and $\delta^{18} \mathrm{O}$ variation in carbonate deposits from mainly non-marine environments. In: Ruiz-Omeñaca, J.I., Piñuela, L. \& García-Ramos, J.C. (Eds.), Comunicaciones del V Congreso del Jurásico de España. Museo del Jurásico de Asturias (MUJA), Colunga, 150.

Silva, R.L., Duarte, L.V., Comas-Rengifo, M.J., Azerêdo, A.C. (2010b) - Top Ibex-Margaritatus (Pliensbachian) series of the Lusitanian Basin (Portugal): sedimentology, events and highresolution correlation. Livre de Résumé, Réunion spécialisée de la Société Géologique de France en hommage of Serge Elmi, Lyon, France, 95-96.

Silva., R.L., Mendonça Filho, J.G., Duarte, L.V., Comas-Rengifo, M.J., Azerêdo, A.C. and Ferreira, R., (2010c) - Organic-rich facies of the top Ibex-Margaritatus zones (Pliensbachian) of the Lusitanian Basin (Portugal): TOC and biomarkers variation. Geochimica et Cosmochimica Acta; 74 (12-S1), A962.

Wilson, R.C.L., Hiscott, R.N., Willis, M.G., Gradstein, F.M. (1989) - The Lusitanian basin of westcentral Portugal: Mesozoic and Tertiary tectonic, stratigraphy, and subsidence history. AAPG Memoir; 46, 341-361.

Wright, V. P. (1985) - Algal marsh deposits from the Upper Jurassic of Portugal. In: Toomey, D. F. \& Nitecki, M. H. (Eds.), Palaeoalgology: Contemporary Research and Applications 1985, 330341. Springer-Verlag, New York.

Wright, V.P., Azerêdo, A.C. (2006) - How relevant is the role of macrophytic vegetation in controlling peritidal carbonate facies?: Clues from the Upper Jurassic of Portugal. Sedimentary Geology; 186, 147-156. 